\title{
Fit to Dance Survey: elements of lifestyle and injury incidence in Chinese dancers
}

Yanan Dang, MSc ${ }^{1,2}$; Yiannis Koutedakis, $\mathrm{PhD}^{1,3}$; Matthew Wyon, $\mathrm{PhD}^{1,4}$

${ }^{1}$ Institute of Human Sciences, University of Wolverhampton, UK

${ }^{2}$ Beijing Dance Academy, Beijing, China

${ }^{3}$ Department of Sport Science, University of Thessaly, Greece

${ }^{4}$ National Institute of Dance Medicine and Science, Walsall, UK

Corresponding author

Prof Matthew Wyon

Institute of Human Sciences,

University of Wolverhampton,

Gorway Rd, Walsall, WS1 3BD, UK

email: $\underline{\text { m.wyon@wlv.ac.uk }}$

\section{Abstract}

The Fit to Dance survey has been conducted a number of times using primarily Western participants and has provided foundation data for other studies. The purpose of the current study was to replicate the Fit to Dance 2 survey focusing on features of health and injuries in pre-professional and professional Chinese dancers of different genres. Results revealed that respondents $(n=1040)$ were from Chinese Folk dance (44.4\%), Chinese Classical Dance (25.6\%), ballet (10.2\%) and contemporary dance (9.8\%). Compared to the Fit to Dance 2 survey, alcohol consumption (29\% vs $82 \% ; p<0.01$ ) and smoking $(13 \%$ vs $21 \%$; $p<0.05)$ were significantly less in Chinese dancers, but a higher percentage reported using weight reducing eating plans ( $57 \%$ vs $23 \%$; $p<0.01$ ) or having psychological issues with food $(27 \%$ vs $24 \%$; $p<0.05)$. Reported injuries in a 12 -month period prior to data collection were significantly lower in the current survey (49\% vs $80 \% ; p<0.01$ ). The type of injury (muscle and joint/ligament) and perceived cause of injury (fatigue, overwork and reoccurrence of an old injury) were the same in both the 
current and previous survey. Mean injury rate for the studied 12-month period ranged from 4.9 injuries per dancer (contemporary) to 3.4 injuries per dancer (Chinese Folk dance) which is comparable to previously reported data on western dance populations. This survey has provided the first comprehensive data on the health and injury incidence of Chinese dancers.

\section{Keywords}

Injury rate, nutrition, psychology, ballet, contemporary, Folk dance 


\section{Introduction}

Most of dance medicine and science research has been conducted on individuals living in Western countries and little is known about the Asian dance community. However, it is known that there are differences between, say, the Chinese and UK general populations in obesity, smoking, alcohol consumption, mental health and physical activity levels (1-3); it is rather unclear whether such variations are also reflected in specific sub-populations such as dancers.

As performing athletes (4), dancers are involved in daily physical activities which have been often linked to incidents of burnout $(5,6)$ and injuries (7). The latter have been studied via different methodologies extending from self-report surveys (8-10) to prospective epidemiological studies (11-13). Injury incidence has been found to range from $40 \%$ to $94 \%$ in professional dancers and $17 \%$ to $90 \%$ in pre-professional and schoollevel dancer student (14), while injury incidence in relation to dance exposure have been reported as 0.16-1.33/1000 hours in professional dancers $(7,15)$ and $0.57-4.71 / 1000$ hours in pre-professional dancers $(16,17)$. Musculoskeletal injuries are the most common followed by joint/ligament, tendon and bone injuries $(9,12,18-20)$. The lower body is the most affected site followed by the lower back, though genres such as break dance demonstrate different injury profiles due to their different movement patterns (21).

As mentioned above, most of the reported data on elements of health and injuries originate from Western countries, though this could be due to an English-language reporting bias in systematic reviews $(7,14,22,23)$. It is also noteworthy that the majority of the available reports on these areas focus on ballet and contemporary/modern dance $(11,19,20,24-36)$, despite the fact that more recent studies have included Irish, street dance, musical theatre and DanceSport dancers $(10,21,37,38)$. Injury and health characteristics of Chinese Classical and Folk dance genres have not been previously reported. Chinese classical dance has a very long history, dating back to ancient Chinese court dances, traditional folk dances and ritual dances and has been influenced by martial arts, Chinese Opera, aesthetic taste, and even Chinese philosophy (39). Chinese Folk 
dance on the other hand represents the diversity of the Chinese nation with different folk rituals from the working class and reflects their lives and struggles. The overall characteristics are singing and dancing, use of props to tell an obvious plot (39).

Therefore, the aim of the present online survey was to a) examine aspects of health and injury in Chinese dancers using a translated version of the Fit to Dance survey and b) compare the generated data with previous Fit to Dance surveys $(9,10)$.

\section{Methods}

Material and procedures

The One Dance UK's "Dancers and Dance Student" questionnaire (9) was adopted for the purposes of this study and it was translated into Chinese by a native Chinese speaker. The Fit to Dance Surveys are the largest national surveys focused on dancers' health and well-being to date $(8,9)$; the 48 -question survey collected information on a number of areas that included their basic information, training and injury history and psychological health. Basic information included sex, age, height and body mass and ethnicity; smoking history, drinking habits, dietary preferences and supplementations, menstrual history and sleeping habits. Their training and contract history, including a breakdown of their week (hours of class, rehearsal, performance, fitness training, etc), days off, the time they spend to warm-up/cool-down. Injury history questions focused on time off, body region, type of injury (muscle, bone, joint/ligament or tendon), perceived cause of injury, type of professional help sort with injury (physician, physiotherapist, osteopath, etc), any help with rehabilitation, who paid for treatment. Psychological health including eating problems, anxiety, depression, self-confidence, coping mechanisms used, use of professional counsellor or psychologist. The questionnaire has been replicated on other dance genres(10) and has provided foundation data for numerous other studies having been cited 40 times. The resulted questionnaire was enriched by a few new questions whilst some of the original questions were modified to make them more applicable to Chinese dance community. For instance, the Chinese Classical Dance, Chinese Folk dance, Dance Sport and hip-hop/breaking were added to question 7 (What is your main dance form?) and the option "Did not take any treatment" was added to questions 
"What type of professional help did you initially have for the injuries?" (question 17). New questions included: "Did you have a dance injury in the last 12 months?"; "If your injuries are long-term, have you carried on dancing?", "Did you have an injury during your entire dance career?", and "How long did you have time off due to a dance injury during the last 12-months?". Injury was defined as "any injury that prevented a dancer from taking a full part in all dance-related activities that would normally be required of them for a period equal to or greater than 24 hours after the injury was sustained"(11, 12). Respondents could complete information (type, body region, environment where injury occurred and severity) for each injury. Severity was defined as the number of days off from dancing with respondents being able to select 1, 23 or more than 3 days; for long-term injuries they could record the weeks affected. Subsequently, the questionnaire was sent out to 30 dancers who were asked to complete the questionnaire and comment on any ambiguities. The provided data were reviewed and minor amendments were made. Ethical approval was granted by the lead author's institution. The final questionnaire was posted online on a secure platform that meets European General Data Protection Regulation (2018) and advertised through a number of Chinese dance-focused social media platforms for a 3-month period closing in June 2018. Informed consent was the first question of the survey and included the inclusion criteria. Respondents were told they did not have to answer every question, that all data was confidential, and their identity was anonymised. Inclusion criteria required respondents to be either in full-time vocational training $(12$ years + ) or be contracted to a professional company (minimum 6-month contract). Positive confirmation of the above needed to be answered before respondents could proceed to the rest of the survey.

\section{Statistical Analyses}

Multi-factorial ANOVA, Chi-squared and Kruskal-Wallis tests were utilized to interrogate the data; independent variables included gender (male and female), dance genre (e.g. Chinese Folk Dance, Chinese Classical Dance, ballet and contemporary) and level (professional and student); dependent variables were injury data. There were a number of genres (jazz, hip-hop/breaking, musical theatre, tap, etc) with only a few respondents 
$(n<10)$ and these were categorized as "other". Percentages were calculated according to the total number of responses for that question as a number of questions allowed respondents to have multiple answers e.g. number of injuries per year, injury site, perceived cause of injury, etc.). The SPSS version 24 (SPSS, Inc., Chicago, IL, USA) was used for the aforementioned analyses and the level for statistical significance was set at $p \leq 0.05$.

\section{Results}

A total of 1040 individuals completed the questionnaire: 871 were dance students (83.7\%) and 169 professional dancers (16.3\%), of whom $82.8 \%$ were females.

Respondents came from 4 main genres; Chinese Folk dance (44.4\%), Chinese Classical Dance (25.6\%), ballet (10.2\%) and contemporary dance (9.8\%). The majority of respondents were students (83.8\%; males $=123$, age $14 \pm 3.07 \mathrm{yrs}$, height $165.5 \pm 5.69 \mathrm{~cm}$, body mass $64.9 \pm 7.49 \mathrm{~kg}, \mathrm{BMI} 20.7 \pm 1.84$; females $=748$, age $14 \pm 2.93 \mathrm{yrs}$, height $165.5 \pm 4.56 \mathrm{~cm}$, body mass $51.8 \pm 5.79 \mathrm{~kg}, \mathrm{BMI} 18.9 \pm 1.89$ ) and most entered full-time training at the ages of either $10-12$ yrs (36.5\%) or 16-18 yrs (34.0\%). Most of our professional dancers (16.2\%; males $=56$, age $19 \pm 6.75 \mathrm{yrs}$, height $177.1 \pm 6.49 \mathrm{~cm}$, body mass $67.2 \pm 8.89 \mathrm{~kg}$, BMI 21.4 \pm 2.51 ; females $=113$, age $19.6 \pm 6.30 \mathrm{yrs}$, height $165.1 \pm 4.93$ $\mathrm{cm}$, body mass $51.5 \pm 5.40 \mathrm{~kg}, \mathrm{BMI} 18.8 \pm 1.92$ ) reported experience of less than 3 years with contracts between eight to 12 months a year (79.9\%) for approximately 35 hours a week. The professional dancers reported that the average work-week involved 6 hours of dance class, 8 hours rehearsals, 6 hours performances, 7 hours body conditioning (e.g. Pilates) and 6 hours strength and fitness training. Initial analyses indicated that "level" (student vs professional) did not provide statistical differences across the dependent variables and therefore the student and professional data were combined.

\section{General health}

About $87 \%$ of respondents over 18 years old reported not smoking and $71 \%$ not drinking alcohol. Also, 57\% reported following a weight reducing regime getting information from media/literature (45.6\%) and friends (31.1\%); a further $21.4 \%$ reported a current eating disorder, whilst $26.7 \%$ admitted psychological issues with eating. Those on nutritional 
supplementation (34.7\%) mainly took multivitamins (67.9\%) and/or calcium (43.7\%). The total of $54 \%$ thought that 8 hours sleep a night is necessary but only $19 \%$ reported achieving this. The majority of student and professional dancers got 6-7 hours a night, with $10.3 \%$ and $12.4 \%$ reporting getting less than 6 hours, respectively. Both groups reported getting one (students $38 \%$, professionals $45 \%$ ) or two (students $51 \%$, professionals $44 \%$ ) days of rest a week. The majority of female participants $(82.2 \%)$ reported that they started menstruating between $12-15$ years of age and $44.9 \%$ reported irregularities with their periods.

Table 1: Dancers reported being injured in last 12 months

\begin{tabular}{ll|cc} 
Dance genre & Gender & $\begin{array}{r}\text { Injured in last 12-month period } \\
\text { Total }\end{array}$ & $\%$ \\
\hline Chinese Classical dance & $\mathrm{M}=43$ & 30 & 69.8 \\
& $\mathrm{~F}=223$ & 110 & 49.3 \\
\hline Ballet & $\mathrm{M}=24$ & 17 & 70.8 \\
& $\mathrm{~F}=82$ & 41 & 50.0 \\
\hline Contemporary & $\mathrm{M}=40$ & 22 & 55.0 \\
& $\mathrm{~F}=62$ & 38 & 61.3 \\
\hline Chinese Folk dance & $\mathrm{M}=48$ & 30 & 62.5 \\
& $\mathrm{~F}=414$ & 181 & 43.7 \\
\hline DanceSport & $\mathrm{M}=8$ & 4 & 50.0 \\
& $\mathrm{~F}=25$ & 12 & 48.0 \\
\hline Other & $\mathrm{M}=6$ & 8 & 61.5 \\
\hline Total & $\mathrm{F}=55$ & 510 & 36.2 \\
\hline
\end{tabular}

Other = Jazz, hip-hop, breaking, musical theatre, tap

Dance injury

The total of $49 \%$ of our respondents reported at least one dance injury in the 12-months prior to data collection, with significantly more male participants reporting injury (total 
$\chi^{2}=.13, p<0.001$ ) (Table 1). There was a significant difference in self-reported injury rate per person between the genres $\left(F_{8,1039}=2.142 ; p<0.05\right)$ but no statistical differences for gender or for the different tissue types of injury (muscle, bone, joint or tendon) (Table 2). Contemporary dance and ballet reported the highest injury rate ( 4.9 and 4.5 injuries per dancer, respectively) followed by DanceSport (4.0 injuries per dancer), Chinese Classical Dance (3.9 injuries per dancer), and Chinese Folk dance (3.4 injuries per dancer). Dance class was the main environment that injuries occurred (64.8\%), followed by rehearsals (58.2\%) and performance (51.8\%).

Body region of injury incidence

The most injured sites for all the participants were knees, ankles, feet, shoulders, neck and lower back (Table 3; the sites with the highest injury incidence are highlighted for each genre). Multivariate analysis of gender and injury rate per site indicated a significant main effect for dance genre $(F=1.255, p=0.036)$ and genre by gender $(F=1.281, p=0.044)$. Between-subject effects noted a significant difference for injury rate for feet $\left(F_{8,1024}=2.388, p=0.015\right)$ and genre by gender for neck $\left(F_{6,1024}=2.388, p=0.05\right)$, upper back $\left(F_{6,1024}=2.388, p=0.034\right)$, lower back $\left(F_{6,1024}=2.388, p=0.024\right)$, ribs $\left(F_{6,1024}=2.388, p=0.05\right)$, and lower legs $\left(F_{6,1024}=2.388, p=0.021\right)$. Post hoc tests noted that contemporary dance had significantly more arm ( $p=0.029)$, neck $(p=0.006)$ injuries than Chinese Classical Dance and significantly higher than Chinese Folk dance for neck $(p<0.001)$, upper back $(p=0.014)$ and lower back $(p=0.011)$. Ballet reported significantly higher injury incidence than Chinese Folk dance for the groin $(p=0.027)$ and feet $(p=0.006)$ (Table 4). 
Table 2: Total injury and tissue injury rate per person for the different genres and gender over 12-month period

\begin{tabular}{ll|lllll}
\hline Dance genre & Gender & \multicolumn{5}{|c}{ Injury rate per person } \\
& & Total & Muscle & Bone & Joint & Tendon \\
\hline Chinese Classical & $\mathrm{M}=43$ & $5.4 \pm 4.39$ & $1.7 \pm 1.49$ & $1.1 \pm 1.05$ & $1.7 \pm 1.55$ & $1.1 \pm 1.06$ \\
dance & $\mathrm{F}=223$ & $3.7 \pm 4.19$ & $1.2 \pm 1.51$ & $.7 \pm 0.83$ & $1.1 \pm 1.39$ & $.7 \pm 0.96$ \\
Ballet & $\mathrm{M}=24$ & $6.4 \pm 5.54$ & $1.9 \pm 1.62$ & $1.4 \pm 1.53$ & $1.8 \pm 1.61$ & $1.3 \pm 1.23$ \\
& $\mathrm{~F}=82$ & $3.9 \pm 4.44$ & $1.1 \pm 1.44$ & $.7 \pm 0.84$ & $1.2 \pm 1.49$ & $.9 \pm 1.07$ \\
Contemporary & $\mathrm{M}=40$ & $4.8 \pm 4.96$ & $1.5 \pm 1.81$ & $.9 \pm 1.19$ & $1.4 \pm 1.57$ & $.9 \pm 1.07$ \\
& $\mathrm{~F}=62$ & $5.1 \pm 4.87$ & $1.7 \pm 1.66$ & $.9 \pm 1.15$ & $1.5 \pm 1.56$ & $.9 \pm 1.07$ \\
Chinese Folk dance & $\mathrm{M}=48$ & $5.3 \pm 4.89$ & $1.6 \pm 1.59$ & $1.1 \pm 1.2$ & $1.6 \pm 1.61$ & $1.1 \pm 1.24$ \\
& $\mathrm{~F}=414$ & $3.2 \pm 4.09$ & $1.1 \pm 1.41$ & $.7 \pm 1.01$ & $.9 \pm 1.27$ & $.6 \pm 0.84$ \\
DanceSport & $\mathrm{M}=8$ & $3.6 \pm 4.31$ & $1.0 \pm 1.21$ & $.9 \pm 1.13$ & $1.0 \pm 1.19$ & $.8 \pm 0.89$ \\
& $\mathrm{~F}=25$ & $1.1 \pm 4.73$ & $1.3 \pm 1.68$ & $.6 \pm 0.71$ & $1.4 \pm 1.61$ & $.8 \pm 1.08$ \\
Other & $\mathrm{M}=16$ & $5.8 \pm 6.09$ & $1.9 \pm 2.02$ & $1.2 \pm 1.59$ & $1.5 \pm 1.56$ & $1.2 \pm 1.41$ \\
& $\mathrm{~F}=55$ & $3.3 \pm 4.82$ & $1.1 \pm 1.63$ & $.7 \pm 1.05$ & $.9 \pm 1.45$ & $.6 \pm 1.04$ \\
\hline Total & & & $1.2 \pm 1.52$ & $.8 \pm 1.02$ & $1.1 \pm 1.42$ & $.7 \pm 0.99$ \\
\hline
\end{tabular}

Other = Jazz, hip-hop, breaking, musical theatre, tap 
Table 3: Percentage of participants that reported injuries at each site*

\begin{tabular}{|c|c|c|c|c|c|c|c|c|c|c|c|c|c|c|c|}
\hline \multirow[t]{2}{*}{ Dance genre } & \multirow[t]{2}{*}{ Gender } & \multicolumn{14}{|c|}{$\%$} \\
\hline & & Arms & Shoulders & Neck & U/Back & L/Back & Ribs & Pelvis & Groin & Hips & U/Leg & Knees & L/Legs & Ankles & Feet \\
\hline Chinese Classical & $M=43$ & 67.4 & 65.12 & 65.12 & 51.2 & 58.1 & 44.2 & 41.9 & 60.5 & 39.5 & 53.5 & 86.1 & 53.5 & 69.8 & 65.1 \\
\hline dance & $F=223$ & 61.9 & 63.6 & 62.33 & 64.6 & 68.2 & 56.1 & 56.9 & 65.0 & 54.7 & 64.1 & 78.0 & 58.7 & 78.9 & 72.7 \\
\hline \multirow[t]{2}{*}{ Ballet } & $M=24$ & 50 & 62.7 & 62.5 & 58.3 & 58.5 & 45.8 & 45.8 & 58.3 & 45.8 & 62.5 & 83.3 & 62.5 & 75 & 75 \\
\hline & $F=82$ & 57.3 & 62.2 & 69.5 & 62.2 & 70.7 & 53.7 & 54.9 & 70.7 & 54.9 & 62.2 & 76.8 & 60.9 & 85.4 & 85.4 \\
\hline \multirow[t]{2}{*}{ Contemporary } & $M=40$ & 85 & 82.5 & 72.5 & 62.5 & 60 & 50 & 55 & 60 & 57.5 & 60 & 75 & 57.5 & 82.5 & 80 \\
\hline & $F=62$ & 62.9 & 67.7 & 70.97 & 66.1 & 66.1 & 51.6 & 46.8 & 56.5 & 48.4 & 58.1 & 74.2 & 50 & 72.6 & 69.4 \\
\hline Chinese Folk & $M=48$ & 68.8 & 66.6 & 64.58 & 62.5 & 62.5 & 56.3 & 60.4 & 66.7 & 56.3 & 66.7 & 85.4 & 68.8 & 81.3 & 79.2 \\
\hline dance & $F=414$ & & 73.9 & 72.22 & 66.4 & 68.3 & 61.8 & 63.1 & 67.2 & 62.6 & 71.3 & 81.6 & 64.5 & 77.8 & 72.7 \\
\hline \multirow[t]{2}{*}{ DanceSport } & $M=8$ & 75 & 62.6 & 62.5 & 62.5 & 62.5 & 75 & 62.5 & 62.5 & 62.5 & 62.5 & 87.5 & 62.5 & 65.5 & 62.5 \\
\hline & $F=25$ & 70.1 & 68 & 76 & 64 & 76 & 60 & 64 & 76 & 60 & 68 & 92 & 72 & 92 & 68 \\
\hline \multirow[t]{2}{*}{ Other } & $M=16$ & 92.3 & 69.3 & 69.23 & 61.5 & 46.4 & 46.2 & 46.2 & 61.5 & 46.2 & 61.5 & 76.9 & 46.2 & 76.9 & 69.2 \\
\hline & $F=55$ & 78.7 & 76.6 & 80.85 & 68.1 & 72.3 & 65.9 & 68.1 & 72.3 & 63.8 & 65.9 & 91.5 & 72.3 & 76.6 & 70.2 \\
\hline Total & & 33.79 & 39.06 & 38.09 & 28.52 & 34.57 & 14.45 & 16.21 & 31.25 & 13.8 & $31 . \varepsilon$ & 61.5 & 23.0 & 56.4 & 546. \\
\hline
\end{tabular}

*The four highest injury sites for each genre and gender are highlighted in bold;

Other = Jazz, hip-hop, breaking, musical theatre, tap;

U/Back=Upper back; L/Back= Lower back; U/Leg= Upper leg; L/Leg= Lower leg 
Table 4: Injury rate for site by genre and gender

\begin{tabular}{|c|c|c|c|c|c|c|c|c|c|c|c|c|c|c|c|}
\hline \multirow[t]{2}{*}{ Dance genre } & \multirow[t]{2}{*}{ Gender } & \multicolumn{14}{|c|}{ Injury rate per person } \\
\hline & & Arms & Shoulders & Neck & U/Back & L/Back & Ribs & Pelvis & Groin & Hips & U/Leg & Knees & L/Legs & Ankles & Feet \\
\hline Chinese Classical & $M=43$ & $1.4 \pm 1.35$ & $1.3 \pm 1.25$ & $1.3 \pm 1.25$ & $1.1 \pm 0.99$ & $1.4 \pm 1.12$ & $.9 \pm 0.76$ & $.9 \pm 0.74$ & $1.1 \pm 1.01$ & $.8 \pm 0.66$ & $1.2 \pm 1.29$ & $1.8 \pm 1.57$ & $1.1 \pm 1.26$ & $1.3 \pm 1.23$ & $1.4 \pm 1.45$ \\
\hline dance & $F=223$ & $.7 \pm 0.88$ & $.7 \pm 0.98$ & $.7 \pm 0.81$ & $.7 \pm 0.96$ & $.8 \pm 1.12$ & $.6 \pm 0.67$ & $.6 \pm 0.74$ & $.8 \pm 1.0$ & $.6 \pm 0.63$ & $.7 \pm 0.89$ & $1.0 \pm 1.35$ & $.6 \pm 0.83$ & $.9 \pm 1.24$ & $.8 \pm 1.07$ \\
\hline \multirow[t]{2}{*}{ Ballet } & $M=24$ & $1.2 \pm 1.21$ & $1.3 \pm 1.11$ & $1.3 \pm 1.31$ & $1.5 \pm 1.56$ & $.9 \pm 1.15$ & $1.0 \pm 0.98$ & $1.0 \pm 0.98$ & $1.5 \pm 1.56$ & $1.0 \pm 0.98$ & $1.3 \pm 1.27$ & $1.7 \pm 1.48$ & $1.3 \pm 1.19$ & $1.5 \pm 1.41$ & $1.6 \pm 1.44$ \\
\hline & $F=82$ & $.7 \pm 0.88$ & $.7 \pm 0.92$ & $.8 \pm 1.09$ & $.7 \pm 0.88$ & $.9 \pm 1.49$ & $.6 \pm 0.71$ & $.6 \pm .71$ & $.9 \pm 1.18$ & $.6 \pm 0.98$ & $.7 \pm 0.96$ & $.9 \pm 1.27$ & $.7 \pm 0.88$ & $1.1 \pm 1.34$ & $1.1 \pm 1.39$ \\
\hline \multirow[t]{2}{*}{ Contemporary } & $M=40$ & $1.3 \pm 1.45$ & $1.2 \pm 1.32$ & $1.2 \pm 1.48$ & $.9 \pm 1.26$ & $1.3 \pm 1.57$ & $.7 \pm 0.91$ & $.7 \pm 0.88$ & $.8 \pm 1.04$ & $.8 \pm 1.02$ & $.9 \pm 1.17$ & $1.4 \pm 1.64$ & $.9 \pm 1.18$ & $1.3 \pm 1.43$ & $1.4 \pm 1.64$ \\
\hline & $F=62$ & $1.1 \pm 1.34$ & $1.2 \pm 1.31$ & $1.3 \pm 1.43$ & $1.1 \pm 1.19$ & $1.3 \pm 1.57$ & $.8 \pm 0.89$ & $.7 \pm 0.77$ & $.9 \pm 0.91$ & $.8 \pm 0.84$ & $1.1 \pm 1.33$ & $1.3 \pm 1.48$ & $.9 \pm 1.03$ & $1.2 \pm 1.27$ & $1.3 \pm 1.45$ \\
\hline \multirow[t]{2}{*}{ Chinese Folk dance } & $M=48$ & $1.2 \pm 1.34$ & $1.2 \pm 1.23$ & $1.2 \pm 1.27$ & $1.1 \pm 1.16$ & $1.1 \pm 1.23$ & $.9 \pm 0.96$ & $1.0 \pm 1.07$ & $1.2 \pm 1.33$ & $1.0 \pm 1.16$ & $1.1 \pm 1.18$ & $1.7 \pm 1.78$ & $1.2 \pm 1.33$ & $1.4 \pm 1.41$ & $1.3 \pm 1.27$ \\
\hline & $F=414$ & $.6 \pm 0.86$ & $.7 \pm 0.99$ & $.7 \pm 0.92$ & $.6 \pm 0.86$ & $.7 \pm 1.04$ & $.5 \pm 0.66$ & $.5 \pm 0.76$ & $.6 \pm 0.85$ & $.5 \pm 0.74$ & $.7 \pm 0.95$ & $.9 \pm 1.31$ & $.6 \pm 0.78$ & $.8 \pm 1.18$ & $.7 \pm 1.02$ \\
\hline \multirow[t]{2}{*}{ DanceSport } & $M=8$ & $.8 \pm 0.89$ & $.6 \pm 0.74$ & $.6 \pm 0.74$ & $.6 \pm 0.74$ & $.6 \pm 0.74$ & $0.3 \pm 1.83$ & $.6 \pm 0.74$ & $.6 \pm 0.74$ & $.6 \pm 0.74$ & $.6 \pm 0.74$ & $1.0 \pm 1.19$ & $.6 \pm 0.74$ & $.6 \pm 0.74$ & $.6 \pm 0.74$ \\
\hline & $F=25$ & $.6 \pm 0.76$ & $.8 \pm 1.28$ & $1.1 \pm 1.51$ & $.6 \pm 0.81$ & $1.1 \pm 1.59$ & $.7 \pm 0.99$ & $.7 \pm 0.89$ & $.8 \pm 1.04$ & $.6 \pm 0.91$ & $.8 \pm 1.19$ & $1.1 \pm 1.29$ & $.9 \pm 1.35$ & $1.1 \pm 1.33$ & $.8 \pm 1.25$ \\
\hline \multirow[t]{2}{*}{ Other } & $M=16$ & $1.2 \pm 1.09$ & $1.0 \pm 1.0$ & $1.0 \pm 1.0$ & $1.0 \pm 1.16$ & $.8 \pm 0.83$ & $.8 \pm 0.95$ & $.9 \pm 1.07$ & $.9 \pm 0.95$ & $.7 \pm 0.63$ & $1.0 \pm 1.19$ & $1.1 \pm 1.04$ & $.9 \pm 1.07$ & $1.3 \pm 1.44$ & $1.0 \pm 1.0$ \\
\hline & $F=55$ & $.7 \pm 1.15$ & $.6 \pm 0.91$ & $.7 \pm 1.28$ & $.5 \pm 0.86$ & $.6 \pm 1.02$ & $.4 \pm 0.53$ & $.4 \pm 0.58$ & $.5 \pm 0.78$ & $.4 \pm 0.49$ & $.4 \pm 0.72$ & $.9 \pm 1.48$ & $.6 \pm 0.87$ & $.6 \pm 1.07$ & $.5 \pm 0.88$ \\
\hline Total & & $.8 \pm 1.03$ & $.8 \pm 1.06$ & $.8 \pm 1.07$ & $.7 \pm 0.98$ & $.9 \pm 1.18$ & $.6 \pm 0.76$ & $.6 \pm 0.79$ & $.8 \pm 0.99$ & $.6 \pm 0.77$ & $.8 \pm 1.02$ & $1.1 \pm 1.4$ & $.7 \pm 0.94$ & $.9 \pm 1.26$ & $.9 \pm 1.19$ \\
\hline
\end{tabular}

Other = Jazz, hip-hop, breaking, musical theatre, tap;

U/Back=Upper back; L/Back= Lower back; U/Leg= Upper leg; L/Leg= Lower leg 
Perceived cause of dance injuries

Respondents reported that the main perceived cause of their injuries were recurrence of old dance injury, fatigue, overwork and insufficient warm up (Table 5). Breakdown by genre and gender indicated no significant differences in the perceived causes of injury; variations included incorrect technique/training (male Chinese traditional and contemporary dance; female Chinese Folk dance; male and female DanceSport); and ignoring early warning signs (female and male ballet).

Injury severity

The total of $38.7 \%$ of our dancers reported having a long-term injury, with $20.8 \%$ indicating they had an injury for between 2-12 months. Though $78 \%$ of respondents reported still being able to dance when injured, Kruskal-Wallis analysis indicated significant differences between the genres for injury severity for class $(p=0.05)$, rehearsal $(p=0.035)$ and performance $(p=0.05)$ (Table 6). Within each category, Chinese Classical and Folk genres had the highest severity followed by ballet and then contemporary dance. 
Table 5: Perceived causes of injury*

\begin{tabular}{|c|c|c|c|c|c|c|c|c|c|c|c|c|c|}
\hline \multirow[t]{2}{*}{ Dance genre } & \multirow[t]{2}{*}{ Gender } & \multirow[b]{2}{*}{ Fatigue } & \multirow[b]{2}{*}{ Overwork } & \multirow[b]{2}{*}{$\begin{array}{l}\text { Unsuitable } \\
\text { floor }\end{array}$} & \multirow[b]{2}{*}{$\begin{array}{c}\text { Cold } \\
\text { environment }\end{array}$} & \multirow[b]{2}{*}{$\begin{array}{l}\text { Insufficient } \\
\text { warm up }\end{array}$} & \multirow[b]{2}{*}{$\begin{array}{l}\text { New/difficult } \\
\text { choreography }\end{array}$} & \multirow[b]{2}{*}{$\begin{array}{l}\text { Different } \\
\text { repertory }\end{array}$} & \multicolumn{3}{|c|}{ Injury causes $(\%)$} & \multirow[b]{2}{*}{$\begin{array}{l}\text { Ignoring } \\
\text { early } \\
\text { warning } \\
\text { signs }\end{array}$} & \multirow[b]{2}{*}{$\begin{array}{c}\text { Recurrence } \\
\text { of old } \\
\text { injury }\end{array}$} \\
\hline & & & & & & & & & $\begin{array}{l}\text { Repetitive } \\
\text { movements }\end{array}$ & $\begin{array}{l}\text { Partnering } \\
\text { work }\end{array}$ & $\begin{array}{c}\text { Incorrect } \\
\text { technique/training }\end{array}$ & & \\
\hline \multirow{2}{*}{$\begin{array}{l}\text { Chinese Classical } \\
\text { dance }\end{array}$} & $\mathrm{M}=43$ & 32.5 & 32.6 & 16.2 & 4.6 & 23.3 & 4.7 & 2.3 & 9.3 & 9.3 & 32.6 & 16.3 & 34.9 \\
\hline & $\mathrm{F}=223$ & 21.5 & 21.9 & 2.7 & 0.5 & 17.5 & 4.9 & 0.5 & 9.4 & 0.5 & 15.3 & 10.8 & 24.2 \\
\hline \multirow[t]{2}{*}{ Ballet } & $\mathrm{M}=24$ & 58.3 & 37.5 & 20.8 & 25 & 20.8 & 16.7 & 0 & 8.3 & 8.3 & 33.3 & 37.5 & 37.5 \\
\hline & $\mathrm{F}=82$ & 30.5 & 26.8 & 10.9 & 3.7 & 18.3 & 10.9 & 4.9 & 6.1 & 2.4 & 15.9 & 20.7 & 24.4 \\
\hline \multirow[t]{2}{*}{ Contemporary } & $\mathrm{M}=40$ & 35 & 32.5 & 15 & 5 & 15 & 10 & 5 & 7.5 & 10 & 12.5 & 10 & 27.5 \\
\hline & $\mathrm{F}=62$ & 35.5 & 35.4 & 12.9 & 8.1 & 19.4 & 19.4 & 9.7 & 16.1 & 6.5 & 22.6 & 19.4 & 37.1 \\
\hline \multirow[t]{2}{*}{ Chinese Folk dance } & $\mathrm{M}=48$ & 20.8 & 22.9 & 14.6 & 4.2 & 16.7 & 8.3 & 6.3 & 8.3 & 6.3 & 20.8 & 16.7 & 33.3 \\
\hline & $\mathrm{F}=414$ & 15.7 & 14.5 & 4.8 & 2.7 & 19.1 & 6.0 & 0.7 & 3.1 & 0.7 & 17.2 & 8.5 & 23.4 \\
\hline \multirow[t]{2}{*}{ DanceSport } & $\mathrm{M}=8$ & 25 & 25 & 0 & 0 & 12.5 & 0 & 0 & 0 & 0 & 25 & 0 & 37.5 \\
\hline & $\mathrm{F}=25$ & 32 & 36 & 12 & 0 & 20 & 0 & 0 & 4 & 16 & 24 & 16 & 40 \\
\hline \multirow[t]{2}{*}{ Other } & $\mathrm{M}=16$ & 23.1 & 30.8 & 23.1 & 15.4 & 15.4 & 0 & 7.7 & 0 & 0 & 23.1 & 7.7 & 23.1 \\
\hline & $\mathrm{F}=55$ & 19.2 & 17.1 & 2.3 & 2.1 & 14.9 & 6.4 & 2.1 & 6.4 & 4.3 & 14.9 & 6.4 & 27.7 \\
\hline Total & & 45.9 & 43.9 & 14.8 & 6.8 & 37.1 & 14.6 & 4.5 & 12.9 & 5.7 & 36.5 & 24.4 & 53.7 \\
\hline
\end{tabular}

Top four perceived causes in bold

Other = Jazz, hip-hop, breaking, musical theatre, tap; 
Table 6: Injury severity: Percentage of respondents reporting days off dance activity because of injury for class, rehearsal and performance

\begin{tabular}{|c|c|c|c|c|c|c|c|c|c|c|c|c|c|}
\hline \multirow[b]{2}{*}{ Genre } & & \multicolumn{4}{|c|}{$\begin{array}{c}\text { Class } \\
\text { Days off injured }\end{array}$} & \multicolumn{4}{|c|}{$\begin{array}{c}\text { Rehearsal } \\
\text { Days off injured }\end{array}$} & \multicolumn{4}{|c|}{$\begin{array}{c}\text { Performance } \\
\text { Days off injured }\end{array}$} \\
\hline & & 1 & 2 & 3 & $3+$ & 1 & 2 & 3 & $3+$ & 1 & 2 & 3 & $3+$ \\
\hline \multirow{2}{*}{$\begin{array}{l}\text { Chinese Classical } \\
\text { dance }\end{array}$} & M & 6.9 & 4.7 & 25.6 & 18.6 & 6.9 & 2.3 & 30.2 & 16.38 & 6.9 & 4.7 & 23.3 & 16.3 \\
\hline & $\mathrm{F}$ & 6.7 & 4.5 & 6.7 & 11.7 & 5.4 & 5.8 & 5.8 & 7.6 & 6.7 & 4.9 & 6.3 & 4.9 \\
\hline \multirow[t]{2}{*}{ Ballet } & $\mathrm{M}$ & 8.3 & 4.2 & 4.2 & 29.2 & 8.3 & 4.2 & 8.3 & 25.0 & 12.5 & 8.3 & 4.2 & 16.7 \\
\hline & $\mathrm{F}$ & 8.5 & 6.1 & 8.5 & 14.6 & 10.9 & 4.9 & 10.9 & 10.9 & 9.8 & 2.4 & 10.9 & 8.5 \\
\hline \multirow{2}{*}{ Contemporary dance } & $\mathrm{M}$ & & 7.5 & 20.0 & 5.0 & 2.5 & 5.0 & 15.0 & 7.5 & & 7.5 & 12.5 & 7.5 \\
\hline & $\mathrm{F}$ & 1.6 & 4.8 & 9.7 & 19.4 & 6.5 & 8.1 & 8.1 & 9.8 & 9.7 & 3.2 & 8.1 & 6.5 \\
\hline \multirow[t]{2}{*}{ Chinese Folk dance } & $\bar{M}$ & 8.3 & 12.5 & 10.4 & 10.4 & 10.4 & 14.6 & 12.5 & 6.3 & 6.3 & 16.7 & 10.4 & 6.3 \\
\hline & $\mathrm{F}$ & 5.1 & 5.1 & 6.0 & 12.1 & 9.4 & 4.8 & 4.8 & 5.1 & 11.1 & 1.9 & 3.4 & 5.1 \\
\hline \multirow[t]{2}{*}{ DanceSport } & $\mathrm{M}$ & & 25.0 & & & & 25.0 & 12.5 & & 12.5 & & 25.0 & \\
\hline & $\mathrm{F}$ & 8.0 & 8.0 & 12.0 & 8.0 & 8.0 & 8.0 & 4.0 & & 8.0 & 8.0 & 4.0 & \\
\hline \multirow[t]{2}{*}{ Other } & $\mathrm{M}$ & & 15.4 & & 15.4 & & 15.4 & & 15.4 & & 15.4 & & 15.4 \\
\hline & $\mathrm{F}$ & 6.4 & 2.1 & 6.4 & 8.5 & 8.5 & & 8.5 & 4.3 & 4.3 & & 4.3 & 4.3 \\
\hline
\end{tabular}

Other = Jazz, hip-hop, breaking, musical theatre, tap; 


\section{Discussion}

To our knowledge, this is the first dance health and injury survey on Chinese dancers and the first from a non-Western country. Respondent numbers were similar to that of the 2005 Fit to Dance 2 survey (9) but, unlike previous reports, the current sample was dominated by Chinese Folk (44.4\%) and traditional (25.6\%) dancers and only about 30\% participated in Western-based dance genres such as ballet (10.2\%), contemporary dance (9.8\%) and DanceSport (3.2\%).

Smoking prevalence in China is approximately $24 \%$ with $62 \%$ of males and only $3.4 \%$ females being classified as ever-smokers (40). Although only $13 \%$ of the current sample of dancers classified themselves as smokers, the gender divide mimicked the national statistics ( $m=68.3 \%, f=7.7 \%)$. This finding contradicts the Fit to Dance 2 data, where the smoking prevalence in dancers was found to be higher than that of the UK general population ( $21 \%$ vs $14.9 \%$ ) (41). In both Chinese and UK dancers, there was a higher

prevalence of smoking in males than females, which is interesting as smoking is often used by dancers as an appetite suppressant (9). Alcohol consumption was lower for Chinese dancers than their general population (29\% vs 62\%) and considerably lower than the UK populations (dancers $82 \%$ vs general population $79 \%$ ) (42). Both the current survey and that of Fit to Dance indicated that male dancers had a higher prevalence of alcohol consumption than their female counterparts (China $20 \%$ vs. $4 \%$; UK $83 \%$ vs. $80 \%$ ). It is worth-noticing the high levels of alcohol consumption by female dancers in Western countries.

It has been suggested that dance activity itself cannot be used as a weight-loss mechanism, as the energy cost of dancing is comparatively low compared with other exercise forms (43). Therefore, other methods are required to maintain or achieve the ideal body shape (44). The Chinese adult population have an average BMI slightly lower than Caucasian populations which is beneficial but have a higher percentage body fat, in particular abdominal fat (45). Chinese respondents reported using weight reducing eating plans (57\% vs $23 \%$ ) and having more psychological issues with food ( $27 \%$ vs $24 \%$ ) compared to UK data. The Fit to Dance 2 survey reported dancers using alcohol and smoking as appetite 
suppressants (9), but in comparison percentages of alcohol use and smoking were low in the current survey. Therefore, it could be that Chinese dancers are using diet suppression in an attempt to meet the Western ecto-mesophormic "image" of a dancer (44).

Nutritional supplementation was higher in the UK (63\%)(9) than in China (35\%); multivitamins (China 68\%, UK 56\%) and calcium (China 44\%, UK 20\%) were reported to be the main supplements. In both the current survey and Fit to Dance 2, media/literature was the main source of nutritional advice (China 46\%, UK 39\%), though in China the next main source was reported to be friends (31\%) whilst in the UK it was company/staff (33\%).

The percentage of dancers reported being injured in the previous 12 -months $(49.2 \%)$ was much lower than the $80+\%$ reported in the UK surveys $(8,9)$. The injuries per dancer found in this study was lower than previously reported in ballet (4.5 vs. 6.8) (11), similar to contemporary dance (4.9 vs 4.0) (46) and higher than Folk dance (3.4 vs. 2.0)(46). We also found that the reoccurrence of an old injury was the main perceived cause of injury (53.7\%); the corresponding data in previous studies were only $29 \%$ (9) or $9 \%$ (10). Fatigue (45.9\%), overwork (43.9\%) and insufficient warm up (37.1\%) were ranked next, though the reported percentage of each perceived cause was greater than that reported in Fit to Dance 2 (31\%, 31\% and 14\%, respectively) (9). Interestingly, ballet, contemporary, jazz and musical theatre (Western-based genres) responses are in line with available data with fatigue, over-work, unsuitable floors and ignoring early warning signs being the main perceived causes of injury (9). However, most of these perceived causes are preventable $(47,48)$. For instance, the prevention of injury reoccurrence through hybrid intervention has also been shown to reduce injury incidence from 4.4 to 2.2 injuries per dancer (12). Nevertheless, this requires a multidisciplinary medical team to support the injured dancer which is often not available outside of international-touring dance companies.

As reported in previous surveys, dance class remains the environment where the respondents were most likely to become injured, followed by rehearsal and performance $(9,49)$. This is in agreement with available data, although classical ballet rehearsals was found to be the safest environment (11). It is expected, however, that dance class should 
be the safest environment as high risk, repetitive movements and work load and rate can be controlled by the teacher; rehearsals and performance have a greater element of uncontrollable risk.

In agreement with previous dance injury surveys, we found that muscles and joints are the most frequently affected tissues $(9,10,49)$. Knees and ankles were the body regions most injured across the studied genres, with the exception of arms and shoulders in male contemporary dancers, which confirms previous data (50). Impact from jumping has been shown to be the main cause of lower limb injury in dance $(29,51,52)$. The observed shoulder and neck injuries for our male dancers (ballet, contemporary, Chinese Classical Dance and Dance Sport) are probably due to lifting movements.

Fatigue has often been linked to injury incidence $(9,12)$; this was echoed by the current survey where fatigue was named as the second main perceived cause of injury. This is supported by the fact that only $19 \%$ of our respondents reported 8 hours of sleep a night and approximately $41 \%$ of them had had only one rest day a week. Insufficient levels of rest and recovery affect muscle recovery and repair processes $(53,54)$ leading to overtraining, impaired performance and increased risk of chronic injury $(5,12,55,56)$.

It is reasonable to assume that the present results may have been influenced by certain methodological limitations. For example, due to the study's observational nature, causality and changes through time cannot be established. Also, although our sample of dancers is one of the largest ever studied in a single occasion, we nevertheless acknowledge that its size may be relatively small considering the entire population of China. Further, the data are self-reported and retrospective thereby potentially limiting its efficacy.

\section{Conclusion}

Chinese Classical and Folk dancers reported a lower injury incidence than other genres. Similarly, relatively less Chinese dancers reported an injury for a 12-months period, although the injuries reported by our ballet and contemporary dancers were analogous to those previously found in UK. Overwork, fatigue and recurrence of the old injury were 
the main reported cause of injury and knees, ankles and feet the main dance injury sites. The present study has provided baseline data on an under-researched population that has highlighted differences with Western dancers.

\section{Acknowledgments}

The authors would like to thank the Chinese Scholarship Council for their financial contribution; and One Dance UK for use of the survey.

\section{References}

1. Zhou B, Stamler J, Dennis B, Moag-Stahlberg A, Okuda N, Robertson C, et al. Nutrient intakes of middle-aged men and women in China, Japan, United Kingdom, and United States in the late 1990s: the INTERMAP study. J Hum Hypertens. 2003;17(9):623.

2. Peto R, Lopez AD. The future worldwide health effects of current smoking patterns. Tobacco and public health: Science and policy. 2004;281(6).

3. Hallal PC, Andersen LB, Bull FC, Guthold R, Haskell W, Ekelund U, et al. Global physical activity levels: surveillance progress, pitfalls, and prospects. The lancet. 2012;380(9838):247-57.

4. Koutedakis Y, Sharp NCC. The Fit and Healthy Dancer. Chichester: John Wiley and Sons; 1999.

5. Koutedakis Y. Burnout in dance: the physiological viewpoint. J Dance Med Sci. 2000;4(4):122-7.

6. Koutedakis $\mathrm{Y}$, Frischknecht R, Vrbová G, Sharp N, Budgett R. Maximal voluntary quadriceps strength patterns in Olympic overtrained athletes. Med Sci Sports Exerc. 1995;27(4):566-72.

7. Allen N, Ribbans W, Nevill AM, Wyon M. Musculoskeletal injuries in dance: a systematic review. International Journal of Physical Medicine \& Rehabilitation. 2014;3(1):1-8.

8. Brinson P, Dick F. Fit to Dance? London: Calouste Gulbenkian Foundation; 1996.

9. Laws H. Fit to Dance 2 - Report of the second national inquiry into dancers' health and injury in the UK. London: Newgate Press; 2005. 
10. Riding-McCabe T, Ambegaonkar J, Redding E, Wyon M. Fit to Dance Survey: a comparison with DanceSport injuries. Medical Problems in Performing Artists. 2014;29(2):102-10.

11. Allen N, Nevill A, Brooks J, Koutedakis Y, Wyon M. Ballet Injuries: Injury incidence and severity over one year. J Orthop Sports Phys Ther. 2012;42(9):781-90.

12. Allen N, Nevill A, Brooks J, Koutedakis Y, Wyon M. The effect of a comprehensive injury audit program on injury incidence in ballet: a 3-year prospective study. Clin J Sport Med. 2013;25(3):373-8.

13. Kenny SJ, Palacios-Derflingher L, Whittaker JL, Emery CA. The influence of injury definition on injury burden in preprofessional ballet and contemporary dancers. J Orthop Sports Phys Ther. 2018;48(3):185-93.

14. Hincapié C, Morton E, Cassidy J. Musculoskeletal Injuries and Pain in Dancers: A Systematic Review Arch Phys Med Rehabil. 2008;89(9):1819-29.

15. Bronner S, McBride C, Gill A. Musculoskeletal injuries in professional modern dancers: a prospective cohort study of 15 years. J Sports Sci. 2018;36(16):1880-8.

16. Kenny SJ, Whittaker JL, Emery CA. Risk factors for musculoskeletal injury in preprofessional dancers: a systematic review. Br J Sports Med. 2015:bjsports-2015095121.

17. Bronner S, Bauer NG. Risk factors for musculoskeletal injury in elite pre-professional modern dancers: A prospective cohort prognostic study. Physical Therapy in Sport. 2018;31:42-51.

18. Riding-McCabe T, Ambegaonkar J, Redding E, Wyon M. Fit to Dance Survey: a comparison with DanceSport injuries. Medical Problems in Performing Artists. 2014;29(2):102-10.

19. Bronner S, Ojofeitimi S, Rose D. Injuries in a modern dance company - effect of comprehensive management on injury incidence and time loss. Am J Sports Med. 2003;31(3):365-73.

20. Gamboa J, Roberts L, Maring J, Fergus A. Injury patterns in elite preprofessional ballet dancers and the utility of screening programs to identify risk characteristics. J Orthop Sports Phys Ther. 2008;38(3):126. 
21. Ojofeitimi S, Bronner S, Woo H. Injury incidence in hip hop dance. Scand J Med Sci Sports. 2012;22(3):347-55.

22. Jacobs CL, Hincapié CA, JD C. Musculoskeletal injuries and pain in dancers: a systematic review update. J Dance Med Sci. 2012;16(2):74-84.

23. Kenny SJ, Whittaker JL, Emery CA. Risk factors for musculoskeletal injury in preprofessional dancers: a systematic review. Br J Sports Med. 2016;50(16):997-1003.

24. Allen N, Nevill A, Brooks J, Wyon M. The impact of a performance profiling system using a three-year prospective injury study. In: Solomon R, Solomon J, editors. 20th International Association for Dance Medicine and Science; 28th-31st October 2010; Birmingham, UK: University of Wolverhampton; 2010. p. 9.

25. Briggs J, McCormack M, Hakim A, Grahame R. Injury and joint hypermobility syndrome in ballet dancers - a 5-year follow-up. Rheumatology (Oxf). 2009;48(12):1613 4.

26. Byhring $S, B \varnothing K$. Musculoskeletal injuries in the Norwegian National Ballet: a prospective cohort study. Scand J Med Sci Sports. 2002;12(6):365-70.

27. Ekegren C, Quested R, Brodrick A. Injuries in pre-professional ballet dancers: Incidence, characteristics and consequences. J Sci Med Sport. 2014;17(3):271-5.

28. Garrick J, Requa R. Ballet injuries: An analysis of epidemiology and financial outcome. Am J Sports Med. 1993;21(4).

29. Hopper LS, Allen N, Wyon M, Alderson JA, Elliott BC, Ackland TR. Dance floor mechanical properties and dancer injuries in a touring professional ballet company. J Sci Med Sport. 2014;17(1):29-33.

30. Khan K, Brown J, Way S, Vass N, Crichton K, Alexander R, et al. Overuse injuries in classical ballet. Sports Med. 1995;19(5):341-57.

31. Liederbach M, Dilgen F, Rose D. Incidence of anterior cruciate ligament injuries among elite ballet and modern dancers: a 5-year prospective study. Am J Sports Med. 2008;36(9):1779-88.

32. Nilsson C, Leanderson J, Wykman A, Strender L. The injury panorama in a Swedish professional ballet company. Knee Surg Sports Traumatol Arthrosc. 2001;9:242-6.

33. Solomon R, Micheli LJ, Solomon J, Kelley T. The 'Cost' of Injuries in a Professional Ballet Company: Anatomy of a Season. Med Probl Perform Artists. 1995;10(1):3-10. 
34. Solomon R, Micheli LJ, Solomon J, Kelley T. The 'Cost' of Injuries in a Professional Ballet Company: A Three-Year Perspective. Med Probl Perform Artists. 1996;11(3):67-74. 35. Solomon R, Solomon J, Micheli LJ, McGray Jr E. The 'Cost' of Injuries in a Professional Ballet Company: A Five-Year Study. Med Probl Perform Artists. 1999;14(4):164-9.

36. Twitchett E, Angioi M, Metsios G, Koutedakis Y, Wyon M. Body composition and ballet injuries: a preliminary study. Med Probl Perform Artists. 2008;September:93-8.

37. Bronner S, Brownstein B. Profile of dance injuries in a Broadway show: a discussion of issues in dance medicine epidemiology. J Orthop Sports Phys Ther. 1997;26(2):87-9.

38. Cahalan R, O'Sullivan K. Injury in professional Irish dancers. J Dance Med Sci. 2013;17(4):150-8.

39. Wang K. The history of Chinese dance: Beijing: Foreign Languages Press; 1985.

40. Liu S, Zhang M, Yang L, Li Y, Wang L, Huang Z, et al. Prevalence and patterns of tobacco smoking among Chinese adult men and women: findings of the 2010 national smoking survey. J Epidemiol Community Health. 2017;71(2):154-61.

41. Windsor-Shellard B, Pullin L, Horton M. Adult smoking habits in the UK: 2017 https://www.ons.gov.uk/peoplepopulationandcommunity/healthandsocialcare/healtha ndlifeexpectancies/bulletins/adultsmokinghabitsingreatbritain/2017: Office for National Statistics; 2018 [

42. WHO. Alcohol consumption: levels and patterns https://www.who.int/substance abuse/publications/global alcohol report/en/2018 [

43. Wyon M, Abt G, Redding E, Head A, Sharp N. Oxygen uptake during of modern dance class, rehearsal and performance. J Strength Cond Res. 2004;18(3):646-9.

44. Liiv H, Wyon M, Jurimae T, Saar M, Maest J, Jurimae J. Anthropometry, somatotypes and aerobic power in ballet, contemporary dance and DanceSport. Medical Problems in Performing Artists. 2013;28(4):207-11.

45. Wang J, Thornton JC, Russell M, Burastero S, Heymsfield S, Pierson Jr RN. Asians have lower body mass index (BMI) but higher percent body fat than do whites: comparisons of anthropometric measurements. The American journal of clinical nutrition. 1994;60(1):238.

46. Echegoyen S, Acuña E, Rodríguez C. Injuries in students of three different dance techniques. Med Probl Perform Artists. 2010;25(2):72. 
47. Wyon M. Towards a new training methodology. In: Brown D, Vos M, editors. Ballet, how and why?: on the role of classical ballet in dance education. Arnhem, Netherlands: ArtEZ Press; 2014. p. 111-8.

48. Soligard T, Myklebust G, Steffen K, Holme I, Silvers H, Bizzini M, et al. Comprehensive warm-up programme to prevent injuries in young female footballers: cluster randomised controlled trial. BMJ. 2008;337:a2469.

49. Baker J, Scott D, Watkins K, Keegan-Turcotte S, Wyon M. Self-reported and reported injury patterns in contemporary dance students. Med Probl Perform Artists. 2010;25:105.

50. Sides SN, Ambegaonkar JP, Caswell SV. High incidence of shoulder injuries in collegiate modern dance students. Athletic Therapy Today. 2009;14(4):43-6.

51. Wilson $\mathrm{M}$, Kwon $\mathrm{Y}-\mathrm{H}$. The role of biomechanics in understanding dance movement: a review. J Dance Med Sci. 2008;12(3):109-16.

52. Liederbach M, Dilgen FE, Rose DJ. Incidence of anterior cruciate ligament injuries among elite ballet and modern dancers: a 5-year prospective study. The American journal of sports medicine. 2008;36(9):1779-88.

53. Nédélec M, Halson S, Abaidia A-E, Ahmaidi S, Dupont G. Stress, sleep and recovery in elite soccer: a critical review of the literature. Sports Med. 2015;45(10):1387-400.

54. Dattilo M, Antunes HKM, Medeiros A, Neto MM, Souza HSd, Tufik S, et al. Sleep and muscle recovery: endocrinological and molecular basis for a new and promising hypothesis. Med Hypotheses. 2011;77(2):220-2.

55. Koutedakis $Y$, Budgett R, Faulmann L. Rest and underperforming elite competitors. $\mathrm{Br}$ J Sports Med. 1990;24(4):248-52.

56. Koutedakis Y, Myszkewycz L, Soulas D, Papapostolou V, Sullivan I, Sharp NC. The effects of rest and subsequent training on selected physiological parameters in professional female classical dancers. Int J Sports Med. 1999;20(6):379-83. 
